\title{
Cambios en el contenido de carbono orgánico del suelo tras el rolado de bosques secos en San Luis (Argentina)
}

\author{
David Somovilla Lumbreras ${ }^{122}$; Ricardo PÁez 2 Esteban G. JobbágY² \& Marcelo D. \\ NOSETTO ${ }^{2,3,1}$, \\ ${ }^{1}$ Departamento de Ciencias Agroforestales de la Escuela Técnica Superior de Ingenierías Agrarias de Palencia, Universidad \\ de Valladolid, España. ${ }^{2}$ Grupo de Estudios Ambientales (IMASL-CONICET Y UNSL), San Luis, Argentina. ${ }^{3}$ Cátedra de \\ Climatología Agrícola (FCA-UNER), Entre Ríos, Argentina.
}

\begin{abstract}
Resumen. El carbono orgánico del suelo (COS) es un componente fundamental del ciclo global del carbono, con influencia en diversos servicios ecosistémicos. Si bien los cambios groseros en la cobertura vegetal, como la deforestación, son capaces de modificar su contenido y dinámica, otros más sutiles también pueden resultar importantes. En este trabajo evaluamos los efectos del rolado (i.e., destrucción mecánica parcial de la vegetación leñosa), una técnica muy usada en los sistemas ganaderos de la región chaqueña, sobre el contenido de COS en bosques secos de la provincia de San Luis. Para ello muestreamos el suelo superficial $(<40 \mathrm{~cm})$ en tres sitios con lotes pareados de bosque nativo y rolado, y analizamos su contenido de carbono orgánico y nitrógeno total. De forma complementaria, cuantificamos la proporción de las distintas coberturas vegetales (i.e., árbol, arbusto y pasto) y analizamos la dinámica del verdor satelital. En promedio, los sitios rolados almacenaron $\sim 5 \mathrm{Mg} / \mathrm{ha}$ más de COS que los sitios de bosque $(+13 \%)$. Los mayores contrastes se encontraron en el suelo superficial $(0-20 \mathrm{~cm})$, donde los rolados superaron a los bosques en hasta $30 \%$, mientras que a mayor profundidad (20-40 $\mathrm{cm})$ no se encontraron diferencias significativas. Los sitios rolados también mostraron mayor contenido de nitrógeno total en el suelo superficial $(0-10 \mathrm{~cm},+20 \%)$. Los sitios de bosque presentaron una mayor cobertura aérea de árboles (y menor de arbustos) que los rolados, pero este parámetro no se relacionó con el contenido de COS. Nuestro estudio provee los primeros resultados sobre los efectos del rolado sobre el COS en la región, sin bien los mecanismos subyacentes aún no son claros. Es fundamental avanzar en dicho sentido a fin de diseñar estrategias de manejo tendientes a aumentar el COS y la sustentabilidad de las actividades agropecuarias en la región.
\end{abstract}

[Palabras clave: COS, Chaco Seco, disturbio, NDVI, servicios ecosistémicos, deforestación]

\begin{abstract}
Changes in soil organic carbon after roller-chopping in dry forests of San Luis (Argentina). Soil organic carbon (SOC) is a fundamental component of the global carbon cycle, with influence on different ecosystem services. Although abrupt changes in vegetation cover, such as deforestation, can modify the stock and dynamics of SOC, other subtler changes may also be of importance. In this work, we evaluate the effects of a technique widely used in rangelands of the Chaco region, such as roller-chopping (i.e., partial mechanical destruction of woody vegetation) on the SOC content in dry forests of San Luis province. For this, we sampled the surface soil $(<40 \mathrm{~cm})$ in three sites with native forest and roller-chopped paired plots and analyzed their contents of organic carbon and total nitrogen. In addition, we quantified the proportion of different vegetation covers (i.e., tree, shrub, and grass) and analyzed the NDVI dynamic. On average, roller-chopped stands stored almost $5 \mathrm{Mg} /$ ha more SOC than dry forests $(+13 \%)$. The greatest contrasts were found in the surface soil $(0-20$ $\mathrm{cm}$ depth), where roller-chopped stands exceeded dry forests by up to $30 \%$, while no significant differences were found deeper in the soil profile $(20-40 \mathrm{~cm}$ depth). Roller-chopped stands also showed higher total nitrogen content in the surface soil (0-10 cm depth, $+20 \%)$. Dry forests stands showed higher aerial coverage of trees (and less of shrubs) than roller-chopped stands, but this parameter was not related to SOC contents. Our study provides the first results about the effects of roller-chopping on SOC in the region, although the mechanisms behind these changes have not been clarified yet. It is essential to move forward in this aspect in order to design management strategies aimed at increasing SOC levels and the sustainability of agricultural activities in the region.
\end{abstract}

[Keywords: SOC, Chaco Seco, disturbance, NDVI, ecosystem services, deforestation]

Editor asociado: Gervasio Piñeiro
Recibido: 4 de Septiembre de 2018

Aceptado: 25 de Dicieubre de 2018 


\section{INTRODUCCIÓN}

Los bosques secos, y en particular los de la Argentina, continúan siendo transformados de forma intensa para incrementar la producción agrícola y ganadera. Este proceso, que en los últimos años se aceleró hasta alcanzar tasas máximas a nivel global (Zak et al. 2004; Houspanossian et al. 2016), genera preocupación por los impactos que puede tener sobre los servicios ecosistémicos que proveen estos ambientes. En este sentido, el almacenaje de carbono orgánico en el suelo (COS) es un aspecto crucial dado que afecta múltiples funciones del ecosistema, desde la provisión de nutrientes y el almacenamiento de agua hasta la regulación climática (Evrendilek et al. 2004; Lal 2004; Powlson et al. 2011). Por lo tanto, es clave comprender los efectos de las trasformaciones más extendidas de los bosques secos de la Argentina sobre el contenido de COS, a fin de anticipar posibles cambios y analizar estrategias de manejo menos agresivas. Entre estas transformaciones, los rolados (i.e., disturbios parciales de la cubierta leñosa para favorecer la producción de pastos) están particularmente extendidos y sus efectos son poco conocidos.

Los bosques secos del hemisferio sur tienen una impronta fundamental en el ciclo global del carbono, incluso mayor que la de los bosques tropicales (Poulter et al. 2014). Los bosques secos del Chaco cubren una superficie mayor a 60 millones de hectáreas, y constituyen la segunda extensión forestal en Sudamérica, luego del Amazonas. A pesar de la transformación intensa que están sufriendo estos bosques, aún presentan una proporción elevada $(\sim 70 \%)$ de su superficie cubierta por vegetación nativa (Houspanossian et al. 2016). Si bien nuevas normativas buscan reducir las altas tasas de deforestación y ordenar la expansión agrícola (Ley Nacional $\mathrm{N}^{\circ}$ 26331, 2007), los bosques siguen estando sometidos a una alta presión de transformación (Camba Sans et al. 2018).

Durante los últimos años, en la Región Chaqueña se expandió la práctica agrícola del rolado con el objetivo de aumentar la receptividad ganadera. Esta práctica consiste en pasar un cilindro metálico lastrado con agua, traccionado por un tractor o topadora, que aplasta los arbustos y los árboles de menor tamaño y deja en pie los árboles de mayor porte. A diferencia de la deforestación tradicional, en la que todos los restos vegetales son quemados, el rolado deja los restos vegetales en el sitio y los semi-incorpora al suelo (Anriquez et al. 2005). El rolado modifica la estructura de la comunidad vegetal al disminuir la cobertura arbórea y arbustiva e incrementar inmediatamente la de pastos (Kunst et al. 2012; Steinaker et al. 2016). No obstante, con el paso del tiempo, estos efectos tienden a diluirse, aumentando la cobertura de arbustos (Steinaker et al. 2016). Los efectos de estas transformaciones sobre una variable clave del ecosistema, como es el contenido de COS, fueron explorados pobremente.

La cantidad de carbono orgánico que se almacena en el suelo de un ecosistema queda determinada por el balance entre la tasa de incorporación de carbono nuevo y la tasa de descomposición del existente. Los cambios en la cobertura vegetal pueden modificar el contenido de $\mathrm{C}$ a través de distintos mecanismos (i.e., la alteración de la cantidad y calidad de los detritos, los cambios en la comunidad microbiana del suelo y las modificaciones en las condiciones microclimáticas [Zogg et al. 1997; Houspanossian et al. 2013; Steinaker et al. 2016; Magliano et al. 2017]). Numerosos trabajos muestran que el reemplazo de bosques por cultivos anuales genera una caída en el contenido de COS (Guo and Gifford 2002; Poeplau et al. 2011), aunque con alta variabilidad según las condiciones climáticas y edáficas entre otros factores. Sin embargo, cuando se los reemplaza por pasturas, los resultados no son tan conclusivos (Murty et al. 2002). Trabajos realizados en la Región Chaqueña no se alejan de dicha tendencia global (Bonino 2006; Conti et al. 2014; Villarino et al. 2017). En cuanto a los efectos del rolado sobre el contenido de COS, el único estudio realizado sugiere que el rolado de un fachinal no produciría cambios significativos luego de tres años de realizado (Anriquez et al. 2005). Estudios satelitales en la región muestran que los sitios rolados presentan una menor productividad aérea y una temperatura superficial mayor que los bosques (Marchesini et al. 2014), lo que podría provocar una caída en el contenido de COS. Sin embargo, el gran ingreso de detritos vegetales que se produce al momento del rolado, al ser semiincorporados al suelo, y conjuntamente con la mayor productividad de raíces típicamente asociada a los pastos (Steinaker and Wilson 2005) podría contrarrestar los efectos antes mencionados.

En este trabajo evaluamos los cambios generados por el rolado, 16 años después de su 
implementación, sobre el contenido superficial de COS y nitrógeno total en la región del Chaco Semiárido de la provincia de San Luis. Para esto, realizamos un muestreo de suelos en tres sitios rolados, con su correspondiente control de bosque no rolado, y analizamos el contenido de COS y de nitrógeno total hasta una profundidad de $40 \mathrm{~cm}$. Además, cuantificamos el porcentaje de cobertura de los distintos tipos de cubierta vegetal (i.e., pastos, arbustos y árboles) y sus efectos sobre el contenido de ambos elementos. Finalmente, analizamos los patrones satelitales del NDVI (del inglés, normalized differences vegetation index) en ambos sistemas como un indicador de la productividad primaria aérea.

\section{MATERIALES y MÉTODOS}

\section{Sitios de estudio}

Los sitios de estudio estuvieron ubicados en el establecimiento "San Bernardo", en el centro de la provincia de San Luis (Argentina), a una altitud de $500 \mathrm{~m} \mathrm{s.} \mathrm{n.} \mathrm{m.} \mathrm{El} \mathrm{área} \mathrm{se} \mathrm{ubica} \mathrm{en} \mathrm{el}$ extremo sur del Chaco Seco, cercano al ecotono con la provincia fitogeográfica del Monte hacia el oeste y con el Espinal hacia el sur (Cabrera 1976). La región presenta una matriz de bosques secos, con parches aislados de otras coberturas vegetales que incluyen pasturas de Cenchrus ciliaris y Eragrostis curvula, lotes rolados $\mathrm{y}$, en menor medida, lotes irrigados de cultivos anuales (e.g., maíz, soja, sorgo) (Marchesini et al. 2013; Steinaker et al. 2016). La cría extensiva de ganado vacuno es la principal actividad económica en la región; en promedio se utilizan cargas animales de 0.05 y $0.2 \mathrm{EV} /$ ha en bosques y pasturas, respectivamente (Ser Beef S.A., comunicación personal). La extracción de leña se restringe al uso de los pobladores locales.

La vegetación leñosa de los bosques está dominada principalmente por dos especies, Prosopis flexuosa y Aspidosperma quebrachoblanco, mientras que el sotobosque está dominado por distintas especies de arbustos como Larrea divaricata y Senna aphylla, y pastos perennes como Stipa eriostachya y Aristida mendocina. Estudios realizados en el establecimiento muestran que la cobertura aérea de Prosopis flexuosa es de $\sim 30 \%$ en los sitios de bosque y de 5\% en los sitios rolados (Marchesini 2011). Le sigue en importancia el arbusto Larrea divaricata, con una cobertura de $27 \%$ en los sitios de bosque y $58 \%$ en los rolados (Marchesini 2011).
El clima de la región es semiárido, con una precipitación media de $360 \mathrm{~mm} /$ año (19672009; Salinas del Bebedero, $15 \mathrm{~km}$ al oeste de los sitios de estudio, y 2009-2017, datos propios en el establecimiento). La temperatura media es $17.8^{\circ} \mathrm{C}$; el mes más cálido (enero) y el más frío (julio) tienen temperaturas medias de 24.8 ${ }^{\circ} \mathrm{C}$ y $10.3{ }^{\circ} \mathrm{C}$, respectivamente. El número de heladas en la región es $\sim 38$ eventos por año; ocurren típicamente entre abril y septiembre (CRU) (New et al. 2002). Los suelos están bien drenados, derivan de depósitos de sedimentos de loess fino a lo largo del Holoceno (Iriondo 1993; Tripaldi et al. 2013) y corresponden principalmente a Torriortentes típicos y Haplustoles énticos. La topografía es suave, con pendientes $<1.5 \%$; la napa freática está a $\sim 30 \mathrm{~m}$ de profundidad.

Para caracterizar los cambios en el COS luego de implementar el rolado seleccionamos tres sitios que incluyeron un lote rolado y su par control de bosque nativo. Ambos lotes en cada sitio estuvieron ubicados en la misma posición topográfica y compartieron el mismo tipo de suelo, lo cual fue confirmado por observación directa a campo. Los rolados se efectuaron en 1998 (16 años al muestreo) mediante la utilización de un rolo de $4 \mathrm{~m}$ de longitud y $1.8 \mathrm{~m}$ de diámetro. Los rolados no fueron sembrados con especies forrajeras ni al momento de su implementación ni posteriormente.

\section{Muestreo y análisis}

La toma de muestras de suelo se realizó sobre una transecta de $90 \mathrm{~m}$ de longitud por lote, cuyo punto inicial y dirección fueron establecidos aleatoriamente. Sobre la transecta se establecieron 15 puntos de muestreo, uno cada $6 \mathrm{~m}$. Para evitar errores sistemáticos, cada punto de muestreo no se estableció en el punto inicial de cada tramo de $6 \mathrm{~m}$, sino que cada tramo se dividió en 6 subtramos (de $1 \mathrm{~m}$ cada uno) y al azar se obtuvo la posición definitiva de cada uno de los puntos de muestreo dentro de la propia transecta. En cada punto de muestreo se registró la cobertura vegetal (árbol, arbusto, pasto) y se tomaron tres submuestras de suelo (0-10, 10-20 y 20-40 cm de profundidad) con la precaución de no alterarlas para calcular posteriormente su densidad aparente. Las submuestras fueron extraídas con un tubo de acero (10 cm de diámetro) que se insertó en el suelo con la ayuda de una masa. 
Todas las submuestras de suelo fueron secadas al aire, tamizadas (malla de $2 \mathrm{~mm}$ ) y pesadas en balanza analítica de precisión. Las 15 submuestras tomadas de cada lote para la profundidad de $0-10 \mathrm{~cm}$ fueron analizadas de manera individual para cuantificar su contenido de carbono y también se las agrupó en una muestra compuesta para determinar posteriormente el contenido de carbono y de nitrógeno. Las submuestras correspondientes a las profundidades de $10-20 \mathrm{~cm}$ y $20-40$ $\mathrm{cm}$ también fueron agrupadas para la determinación de carbono y nitrógeno. En total se analizaron 90 submuestras correspondientes a la profundidad $0-10 \mathrm{~cm}$ ( 3 lotes de bosque $y$ 3 de rolado; 15 puntos por lote) para carbono y 18 muestras agrupadas (3 profundidades x 3 sitios x 2 tratamientos) para carbono y nitrógeno. El análisis de la submuestra se realizó para evaluar el efecto de la cobertura vegetal (árbol, pasto, arbusto) sobre el contenido superficial de COS. Por otro lado, el agrupamiento se decidió por una cuestión logística y de costo de los posteriores análisis. Para determinar la densidad aparente se tomó una alícuota de cada submuestra secada al aire; esta submuestra se sometió a estufa (105 ${ }^{\circ} \mathrm{C}$ ) hasta alcanzar un peso constante. Previo a la determinación de carbono, las submuestras y las muestras agrupadas fueron tratadas con ácido clorhídrico $(0.5 \mathrm{~N})$ durante 1.5 horas y posteriormente lavadas tres veces a fin de eliminar los carbonatos presentes en las muestras (Collins et al. 1999). La determinación de carbono orgánico se realizó mediante combustión seca con un equipo analizador automático de carbono LECO, modelo CR12. A las 18 muestras agrupadas se les determinó el contenido de nitrógeno total mediante el método de oxidación húmeda de Kjeldahl.
A fin de caracterizar la productividad primaria en las áreas de bosque y roladas, se utilizó el NDVI (del inglés normalized difference vegetation index) provisto por el sensor MODIS montado en los satélites Terra y Aqua. Diversos trabajos demostraron que este índice está muy asociado con la productividad primaria neta y con el área foliar (Paruelo et al. 1998; Vassallo et al. 2013). Utilizamos el producto MOD13Q1, que posee una integración temporal de 16 días y una resolución espacial de $250 \mathrm{~m}$. Se seleccionaron 14 lotes en total ( 7 bosques y 7 rolados), dentro de los cuales se encontraban los 6 lotes muestreados. El período de análisis fue 9 años (2001-2009) y las imágenes fueron obtenidas del Oak Ridge National Laboratory (modis.ornl.gov). Las diferencias en NDVI entre rolado y bosque, como así también las diferencias en los contenidos de carbono y nitrógeno, se analizaron mediante pruebas de $t$ pareadas. Por otro lado, el efecto de la cobertura del suelo (pasto, arbusto, árbol) sobre el contenido del COS superficial (0-10 $\mathrm{cm})$ se analizó mediante un ANOVA.

\section{RESULTADOS Y DiscusióN}

El contenido de COS almacenado hasta los $40 \mathrm{~cm}$ de profundidad fue significativamente superior en los sitios rolados. En promedio, los sitios rolados presentaron casi $5 \mathrm{Mg}$ más de carbono orgánico por hectárea $(+13 \%)$ que los sitios de bosque nativo (Figura $1 \mathrm{~A}, P=0.02$ ). Las diferencias entre ambas coberturas oscilaron entre 3.7 y $6.2 \mathrm{Mg} / \mathrm{ha}(+8 \%$ a $+20 \%)$, siempre a favor de los rolados.

Esta constituye una de las pocas estimaciones de los efectos del rolado sobre el contenido de COS en la región del Chaco Seco. En oposición

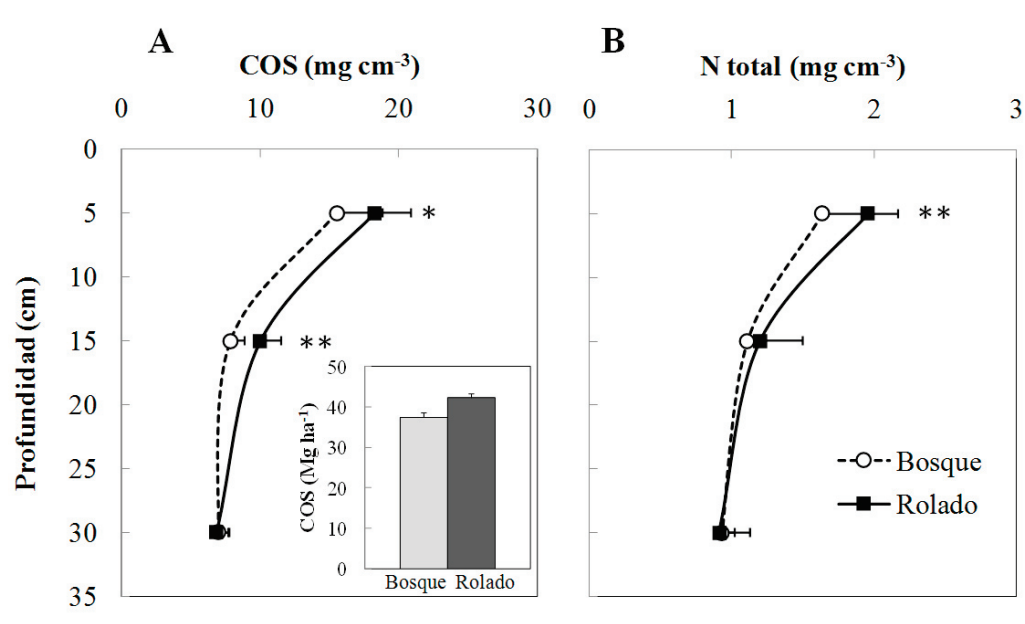

Figura 1. Perfil de carbono orgánico del suelo (COS) (A) y $\mathrm{N}$ total (B) en función de la 3 profundidad en lotes de bosques nativos y rolados. Se reportan los valores medio y error estándar $(n=6)$. Diferencias significativas se indican con * $(\alpha=0.10)$ o ** $(\alpha=0.05)$. La gráfica inserta en el panel A muestra el contenido total de COS acumulado.

Figure 1. Soil Organic Carbon (SOC) (A) and total N (B) variations with soil depth in native forests and roller-chopped stands. Average values and standard error $(n=6)$ are reported. Significant differences are indicated with * $(\alpha=0.10)$ or $* *(\alpha=0.05)$. The inset graphic in panel A shows the total accumulated SOC content. 
a varios estudios en los que se evaluó el efecto del desmonte total (Bonino 2006; Conti et al. 2014; Villarino et al. 2017), nuestro trabajo sugiere que el rolado produce un aumento significativo en el COS superficial tras 16 años desde su implementación. El único estudio previo que evaluó los efectos del rolado se realizó luego de sólo 3 años desde su implementación y no detectó cambios (Anriquez et al. 2005). El momento de observación es una cuestión especialmente relevante en el caso de los rolados, ya que los aportes instantáneos de biomasa muerta aérea y subterránea al suelo son seguidos por procesos más lentos de descomposición, humificación y sucesión ecológica, que tienen lugar en plazos de años a décadas. Los estudios de cronosecuencias o, aun mejor, los seguimientos de largo plazo serían ideales para abordar en más detalle la dinámica temporal del COS.

Los sitios rolados presentaron mayor concentración de carbono orgánico en los horizontes más superficiales del suelo. En los primeros $10 \mathrm{~cm}$ del perfil edáfico, los sitios rolados presentaron $18 \%$ más de carbono almacenado que los sitios de bosque y casi un 30\% más en el horizonte de 10-20 cm de profundidad (Figura 1a). Por otro lado, en el horizonte edáfico más profundo muestreado (20-40 cm) no se observaron diferencias significativas $(P>0.10)$. En el caso de nitrógeno total, sólo se observaron diferencias significativas en el horizonte superficial, donde los sitios rolados mostraron una concentración $20 \%$ superior a los sitios de bosque (Figura 1 b). La relación $\mathrm{C} / \mathrm{N}$ y la densidad aparente del suelo no mostraron diferencias significativas

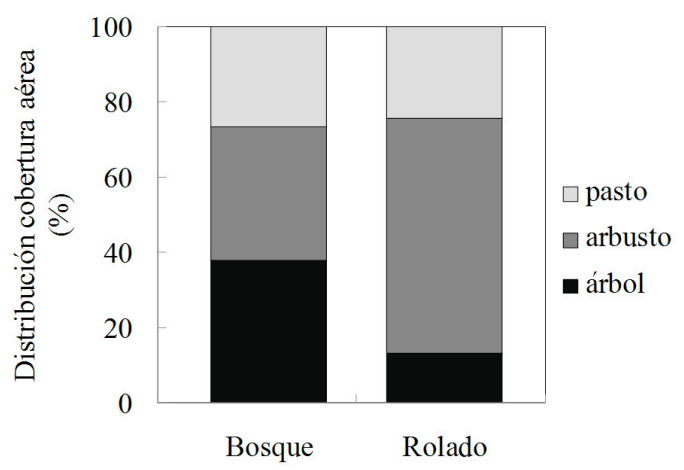

Figura 2. Distribución porcentual promedio de cobertura aérea en lotes de bosques nativos y rolados $(n=6)$.

Figure 2. Average percentage distribution of aerial coverage in native forests and roller-chopped stands $(n=6)$. entre ambas situaciones evaluadas (datos no mostrados).

Los cambios en los efectos del rolado a lo largo del perfil del suelo permiten inferir acerca de los posibles mecanismos involucrados en los patrones observados. Los factores y procesos que explican los cambios de COS son muchos y diversos; los más importantes son los cambios en la tasa de aporte de materia orgánica y las modificaciones en las tasas de descomposición debido a cambios ambientales (Poeplau et al. 2011). El hecho de observar cambios sólo en el suelo superficial, pero no profundo, sugeriría un mayor aporte de detritos orgánicos únicamente en dicha fracción de suelo, dado que si el mayor aporte se debiera a una mayor productividad subterránea (y posiblemente aérea) se observarían cambios a mayor profundidad. Por ejemplo, el aumento de SOC que se suele observar cuando las pasturas reemplazan a los cultivos agrícolas se registra hasta profundidades mayores al metro (Guo and Gifford 2002). Por otro lado, una menor tasa de descomposición por modificaciones en las condiciones ambientales luego del rolado también podría generar el patrón observado. El aumento de temperatura superficial (de hasta $4{ }^{\circ} \mathrm{C}$ ) que se observa en sitios rolados (Marchesini et al. 2014) estaría en contraposición con esta hipótesis. Sin embargo, la fuerte disminución de la humedad edáfica (hasta 20\%) que genera el rolado (Marchesini et al. 2013), que resulta del lavado de sales y del secado más exhaustivo por la absorción vegetal, la avalaría, sobre todo si se tiene en cuenta que esta variable es clave explicando las tasas de descomposición de la materia orgánica en sistemas semiáridos (Garten et al. 2009; Zhou et al. 2012; García et al. 2017). Es posible que la combinación de menores tasas de descomposición y el gran ingreso de biomasa muerta aérea y subterránea resultante del rolado sean parte de la explicación del patrón observado.

Luego de transcurridos 16 años desde el rolado, las dos condiciones evaluadas presentaron claras diferencias en los porcentajes de cobertura de las distintas formas vegetales (Figura 2). Los sitios de bosque presentaron mayor cobertura aérea de árboles respecto a los sitios rolados (38\% vs. $13 \%, P<0.10)$. Pero, por otro lado, los sitios rolados presentaron mayor cobertura de arbustos (62\% vs. $36 \%, P<0.10)$. No se encontraron diferencias en la cobertura de pastos entre ambas condiciones. Tampoco se 

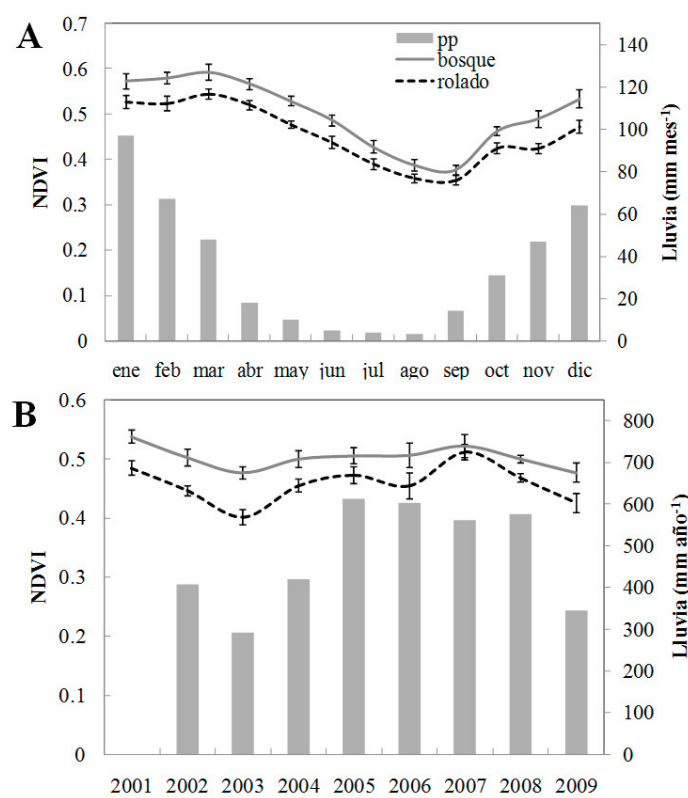

Figura 3. Dinámica estacional (A) e interanual (B) del NDVI en lotes de bosque nativos y rolados. Se reporta el valor medio y el error estándar $(n=14)$. Las columnas indican los valores de lluvia para el área de estudio.

Figure 3. Seasonal (A) and inter-annual (B) NDVI dynamics in native forests and roller-chopped stands. Average values and standard error $(n=6)$ are reported. Columns indicate rainfall amounts for the study region.

observó un efecto de la cobertura aérea sobre el contenido de COS $(P>0.10)$.

Los efectos de largo plazo del rolado sobre la composición de la comunidad vegetal serían negativos desde el punto de vista de su aprovechamiento forrajero. Si bien trabajos previos muestran que la biomasa de pastos y la receptividad ganadera aumentan de manera notable (Steinaker et al. 2016) en plazos cortos, nuestro estudio sugiere que dicho efecto beneficioso parece desaparecer en períodos más extendidos. Agravando esto, la recuperación de la cobertura leñosa no es generada por especies con valor forrajero como Prosopis flexuosa, sino por arbustos no palatables para el ganado como Larrea sp., con un consecuente empobrecimiento del sistema.

La media anual del NDVI, un indicador de la productividad primaria aérea, fue ligeramente superior en los sitios de bosque. En promedio para los 9 años evaluados, el NDVI de los bosques fue $10 \%$ superior a los sitios rolados, pero las diferencias en los valores anuales fluctuaron entre $2 \%$ y $19 \%$. Los mayores contrastes se observaron en años con lluvias por debajo de lo normal (e.g., 2003 y
2009), cuando los rolados acusaron un mayor impacto de la sequía que los bosques (Figura 3a). A nivel intra-anual, ambas coberturas presentaron similar dinámica con valores mínimos en agosto-setiembre y máximos durante el verano (Figura 3b).

En el largo plazo, el rolado causa una reducción en la productividad primaria del ecosistema. Trabajos en la región muestran que el rolado aumenta la productividad de pastos (Kunst et al. 2001; Steinaker et al. 2016); sin embargo, nuestros datos de NDVI sugieren que la productividad total aérea del ecosistema se reduce, lo cual confirma lo observado por otros autores inmediatamente después de la intervención (Marchesini et al. 2014). Es interesante notar que a pesar de haber transcurrido más de diez años de producido el rolado, los valores de NDVI no alcanzaron los niveles pre-rolado. Como lo sugieren otros autores (Steinaker et al. 2016), es probable que la perdida de especies (e.g., Prosopis flexuosa) y grupos funcionales claves (e.g., raíces profundas, fijadoras de nitrógeno) sea la causa de la caída observada de productividad.

Los bosques secos del mundo, y en particular los de Sudamérica, están experimentando fuertes transformaciones, entre las cuales el rolado es una de las más difundidas. Comprender sus efectos sobre la provisión de servicios ecosistémicos, como el almacenamiento de COS, es relevante dada la expansión potencial de esta actividad en más de 40 millones $\mathrm{km}^{2}$ sólo en la Región Chaqueña (Houspanossian et al. 2016). Los resultados, un tanto inesperados, obtenidos en este trabajo demandan trabajos específicos a fin esclarecer los mecanismos responsables de los patrones que se observaron, como así también las tendencias de más largo plazo. Dentro de éstos, cuantificar las tasas de descomposición y sus controles, como así también caracterizar los aportes de detritos orgánicos en cuanto a su volumen, temporalidad y calidad serían fundamentales para avanzar en dicho sentido. Esto es clave para diseñar estrategias de manejo tendientes a aumentar los niveles de COS y la sustentabilidad de las actividades agropecuarias en la región.

Agradecimientos. Este trabajo fue financiado parcialmente por la ANPCyT (PICT 2790/14, Argentina), el CONICET (PIP112-201501-00609, Argentina) y por el International Development Research Center (IDRC 106601-001, Canadá). Agradecemos 
a SER BEEF S.A. por permitirnos acceder a su establecimiento para realizar este trabajo y por la información provista. Agradecemos también a la Universidad de Valladolid (España) y a la Universidad de San Luis
(Argentina) quienes proveyeron una Beca de Intercambio a D. Somovilla para realizar su estancia en la Argentina. También agradecemos a dos revisores anónimos por sus valiosos comentarios.

\section{REFERENCIAS}

Anriquez, A., A. Albanesi, C. Kunst, R. Ledesma, C. López, A. Rodríguez Torresi, and J. Godoy. 2005. Rolado de fachinales y calidad de suelos en el Chaco occidental, Argentina. Ciencia del Suelo 23:145-157.

Bonino, E. E. 2006. Changes in carbon pools associated with a land-use gradient in the Dry Chaco, Argentina. Forest Ecology and Management 223:183-189.

Camba Sans, G. H., S. Aguiar, M. Vallejos, and J. M. Paruelo. 2018. Assessing the effectiveness of a land zoning policy in the Dry Chaco. The Case of Santiago del Estero, Argentina. Land Use Policy 70:313-321.

Collins, H. P., D. R. Christenson, R. L. Blevins, L. G. Bundy, W. A. Dick, D. R. Huggins, and E. A. Paul. 1999. Soil Carbon Dynamics in Corn-Based Agroecosystems: Results from Carbon-13 Natural Abundance. Soil Science Society of America Journal 63:584-591.

Conti, G., N. Pérez-Harguindeguy, F. Quètier, L. D. Gorné, P. Jaureguiberry, G. A. Bertone, L. Enrico, A. Cuchietti, and S. Díaz. 2014. Large changes in carbon storage under different land-use regimes in subtropical seasonally dry forests of southern South America. Agriculture, Ecosystems and Environment 197:68-76.

Evrendilek, F., I. Celik, and S. Kilic. 2004. Changes in soil organic carbon and other physical soil properties along adjacent Mediterranean forest, grassland, and cropland ecosystems in Turkey. Journal of Arid Environments 59:743-752.

García, A. G., C. M. Di Bella, J. Houspanossian, P. N. Magliano, E. G. Jobbágy, G. Posse, R. J. Fernández, and M. D. Nosetto. 2017. Patterns and controls of carbon dioxide and water vapor fluxes in a dry forest of central Argentina. Agricultural and Forest Meteorology 247:520-532.

Garten, C. T., A. T. Classen, and R. J. Norby. 2009. Soil moisture surpasses elevated CO2 and temperature as a control on soil carbon dynamics in a multi-factor climate change experiment. Plant and Soil 319:85-94.

Guo, L. B., and R. M. Gifford. 2002. Soil carbon stocks and land use change: a meta analysis. Global Change Biology 8:345-360.

Houspanossian, J., R. Giménez, G. Baldi, and M. Nosetto. 2016. Is aridity restricting deforestation and land uses in the South American Dry Chaco? Journal of Land Use Science 11:369-383.

Houspanossian, J., M. Nosetto, and E. G. Jobbágy. 2013. Radiation budget changes with dry forest clearing in temperate Argentina. Global Change Biology 19:1211-1222.

Iriondo, M. 1993. Geomorphology and late quaternary of the Chaco (South America). Geomorphology 7:289-303.

Kunst, C., M. Cornacchione, and J. Godoy. 2001. Prácticas de control del fachinal I: Efectos sobre la oferta de forraje de herbáceas. 1er Congreso Nac. sobre Manejo de Pasturas Naturales. Asoc. Manejo de Pastizales Naturales, San Cristóbal, Santa Fe.

Kunst, C., R. Ledesma, S. Bravo, A. Albanesi, A. Anriquez, H. van Meer, and J. Godoy. 2012. Disrupting woody steady states in the Chaco region (Argentina): Responses to combined disturbance treatments. Ecological Engineering 42: $42-53$.

Lal, R. 2004. Soil Carbon Sequestration Impacts on Global Climate Change and Food Security. Science 304:16231627.

Magliano, P. N., R. Fernández, E. L. Florio, F. Murray, and E. G. Jobbágy. 2017. Soil Physical Changes After Conversion of Woodlands to Pastures in Dry Chaco Rangelands (Argentina). Rangeland Ecology and Management 70:225-229.

Marchesini, V., R. J. Fernández, and E. G. Jobbágy. 2013. Salt leaching leads to drier soils in disturbed semiarid woodlands of central Argentina. Oecologia 171:1003-1012.

Marchesini, V. A. 2011. Cambios en el uso de la tierra y el balance de agua en ecosistemas semiáridos: el desmonte selectivo en el Chaco árido analizado a diferentes escalas espaciales. Universidad de Buenos Aires, Buenos Aires.

Marchesini, V. A., R. J. Fernández, J. F. Reynolds, J. A. Sobrino, and C. M. Di Bella. 2014. Changes in evapotranspiration and phenology as consequences of shrub removal in dry forests of central Argentina. Ecohydrology. DOI: 10.1002/ eco.1583.

Murty, D., M. U. F. Kirschbaum, R. E. Mcmurtrie, and H. Mcgilvray. 2002. Does conversion of forest to agricultural land change soil carbon and nitrogen? a review of the literature. Global Change Biology 8:105-123.

New, M., D. Lister, M. Hulme, and I. Makin. 2002. A high-resolution data set of surface climate over global land areas. Climate Research 21:1-25.

Paruelo, J. M., A. B. Beltrán, E. G. Jobbágy, O. E. Sala, and R. A. Golluscio. 1998. The climate of Patagonia: general patterns and controls on biotic processes. Ecología Austral 8:85-101.

Poeplau, C., A. Don, L. Vesterdal, J. Leifeld, B. Van Wesemael, J. Schumacher, and A. Gensior. 2011. Temporal dynamics of soil organic carbon after landロuse change in the temperate zone - carbon response functions as a model approach. Global Change Biology 17:2415-2427.

Poulter, B., D. Frank, P. Ciais, R. B. Myneni, N. Andela, J. Bi, G. Broquet, J. G. Canadell, F. Chevallier, Y. Y. Liu, S. W. Running, S. Sitch, and G. R. van der Werf. 2014. Contribution of semi-arid ecosystems to interannual variability of the global carbon cycle. Nature 509:600-603. 
Powlson, D. S., P. J. Gregory, W. R. Whalley, J. N. Quinton, D. W. Hopkins, A. P. Whitmore, P. R. Hirsch, and K. W. T. Goulding. 2011. Soil management in relation to sustainable agriculture and ecosystem services. Food Policy 36: S72-S87.

Steinaker, D., and S. Wilson. 2005. Belowground litter contributions to nitrogen cycling at a northern grassland-forest boundary. Ecology 86:2825-2833.

Steinaker, D. F., E. G. Jobbágy, J. P. Martini, D. N. Arroyo, J. L. Pacheco, and V. A. Marchesini. 2016. Vegetation composition and structure changes following roller-chopping deforestation in central Argentina woodlands. Journal of Arid Environments 133:19-24.

Tripaldi, A., M. A. Zárate, S. L. Forman, T. Badger, M. E. Doyle, and P. Ciccioli. 2013. Geological evidence for a drought episode in the western Pampas (Argentina, South America) during the early-mid 20th century. The Holocene 23: 1731-1746.

Vassallo, M. M., H. D. Dieguez, M. F. Garbulsky, E. G. Jobbágy, and J. M. Paruelo. 2013. Grassland afforestation impact on primary productivity: a remote sensing approach. Applied Vegetation Science 16:390-403.

Villarino, S. H., G. A. Studdert, P. Baldassini, M. G. Cendoya, L. Ciuffoli, M. Mastrángelo, and G. Piñeiro. 2017. Deforestation impacts on soil organic carbon stocks in the Semiarid Chaco Region, Argentina. Science of the Total Environment 575:1056-1065.

Zak, M. R., M. Cabido, and J. G. Hodgson. 2004. Do subtropical seasonal forests in the Gran Chaco, Argentina, have a future? Biological Conservation 120:589-598.

Zhou, X., C. Chen, Y. Wang, Z. Xu, Z. Hu, X. Cui, and Y. Hao. 2012. Effects of warming and increased precipitation on soil carbon mineralization in an Inner Mongolian grassland after 6 years of treatments. Biology and Fertility of Soils 48:859-866.

Zogg, G. P., D. R. Zak, D. B. Ringelberg, D. C. White, N. W. MacDonald, and K. S. Pregitzer. 1997. Compositional and Functional Shifts in Microbial Communities Due to Soil Warming. Soil Science Society of America Journal 61: 475-481. 